\title{
Pore Shape Modification of a Microporous Metal-Organic Framework Using High Pressure: Accessing a New Phase with Oversized Guest Molecules
}

\author{
Scott C. McKellar, ${ }^{\dagger}$ Jorge Sotelo, ${ }^{\dagger}$ Alex Greenaway, ${ }^{\dagger}$ John P. S. Mowat, ${ }^{\dagger}$ Odin Kvam, ${ }^{\dagger}$ Carole A. Morri- \\ son,,$^{\dagger}$ Paul A. Wright ${ }^{\dagger}$ and Stephen A. Moggach* $*^{\dagger}$ \\ "EaStCHEM School of Chemistry and the Centre for Science at Extreme Conditions, University of Edinburgh, Kings Build- \\ ings, West Mains Road, Edinburgh, EH9 3JJ, UK \\ "EaStCHEM School of Chemistry, University of St Andrews, Purdie Building, St Andrews, KY16 9ST, UK
}

\begin{abstract}
Pressures up to $0.8 \mathrm{GPa}$ have been used to squeeze a range of sterically 'oversized' C5-C8 alkane guest molecules into the cavities of a small-pore Sc-based metal-organic framework. Guest inclusion causes a pronounced reorientation of the aromatic rings of one third of the terephthalate linkers, which act as 'torsion springs', resulting in a fully reversible change in the local pore structure. The study demonstrates how pressure-induced guest uptake can be used to investigate framework flexibility relevant to 'breathing' behavior and to understand the uptake of guest molecules in MOFs relevant to hydrocarbon separation.
\end{abstract}

\section{INTRODUCTION}

One of the major drivers for the current intensive research into porous metal organic frameworks (MOFs) is the potential they offer for applications in gas separation. ${ }^{1}$ The wide variety of chemical environments, pore sizes and shapes, and the flexible behaviour found in many MOFs may improve current separation technologies, or enable previously unfeasible separations. ${ }^{2}$ For example, a recent study has shown that the framework $\mathrm{Fe}_{2} \mathrm{BDP}_{3}$ (BDP = 1,4-benzenedipyrazolate) can separate isomers of hexane according to their degree of branching ${ }^{3}$, which has potential application in gasoline production, where the separation of $\mathrm{C}_{6}$ isomers is a crucial step. ${ }^{3}$ Notably, the triangular channel topology of $\mathrm{Fe}_{2} \mathrm{BDP}_{3}$, which imparts the selectivity, is not observed in zeolites.

Understanding the controlling role of the MOF structure on the kinetics and selectivity of gas uptake is crucial for exploitation in such separation technologies. ${ }^{4}$ Molecular simulations are an important part of this process as they offer insight into the gas adsorption sites and diffusivity through a framework. ${ }^{5,6}$ However, it is becoming increasingly clear that it is not only the framework geometry that determines the uptake behaviour, but also the dynamics and structural response of a framework in the presence of adsorbates. In particular, ligands in MOFs have been observed to show rotation and tilting around an axis and the ability to hinge at the points of their connection to metal cations, all in response to uptake of adsorbates. This can lead to gate opening without change in the time-averaged structure, to subtle distortions and tilts of the framework structure and even to breathing behaviour with very large changes in the pore volumes, sometimes exceeding $100 \%$. $^{7,8}$ To predict adsorption behaviour for any given MOF, for example by molecular simulation, the nature and energetics of its structural response must first be measured and quantified. ${ }^{9,10,11-13}$
Structural changes of these types have been observed over a wide range of conditions in cases where significant guest uptakes occur, ranging from low temperatures and pressures ${ }^{14}$ to room temperature and very high pressures. ${ }^{15,16}$ At very high pressures, the $P \Delta V$ energy term associated with the ingress of molecules from fluids into pores becomes comparable with typical energies of adsorption. For example, for ZIF-8, $\left(\mathrm{Zn}(\mathrm{MeIM})_{2} ; \mathrm{MeIM}=2\right.$-methylimidazolate; $\mathrm{ZIF}=$ zeolitic imidazolate framework) a similar structural response has been observed for different adsorbates under very different conditions. The structure is observed by X-ray diffraction to undergo a transition involving a rotation of the MeIm linkers which increases the porosity of the framework, allowing more guest molecules to enter the pores. This occurs at 0.02 bar in nitrogen gas at 77 $\mathrm{K}$ and also at 1,5 $\mathrm{GPa}$ in liquid methanol at $298 \mathrm{~K}$. The latter was observed for a single crystal of ZIF-8 loaded into a diamond anvil cell (DAC) and surrounded with methanol as a pressuretransmitting liquid (to ensure pressure is applied hydrostatically to the sample) ${ }^{14}$ 17-19 The high-pressure structure was used to model the ambient-pressure structural changes responsible for the increased $\mathrm{N}_{2}$ uptake. ${ }^{14}$ Therefore, as well as directly giving information on the structural response of the MOF to high adsorbate pressures - itself relevant to potential applications of MOFs in high pressure applications such as ultrahigh pressure chromatography, UPLC, or gas storage $\mathrm{e}^{11-13}$ - such diamond anvil studies can shed light on the structural behaviour (adsorption site energies; flexibility) during adsorption at lower pressures. Like the imidazolate ZIF-8, the scandium 1,4-benzenedicarboxylate $\mathrm{Sc}_{2} \mathrm{BDC}_{3}$ is a highly stable $3 \mathrm{D}$-connected small pore MOF. ${ }^{20}$ The structure comprises isolated $\mathrm{ScO}_{6}$ octahedra, all $\mathrm{O}$ atoms of which are part of linking BDC ligands (see Figure 1a). The framework possesses one-dimensional channels with a triangular cross-section $\sim 4 \AA$ in free diameter. Adsorption isotherms show that the framework adsorbs small fuel-related molecules, namely $\mathrm{CO}_{2}$ (kinetic diameter $\sim 3 \AA$ ) and normal alkanes 

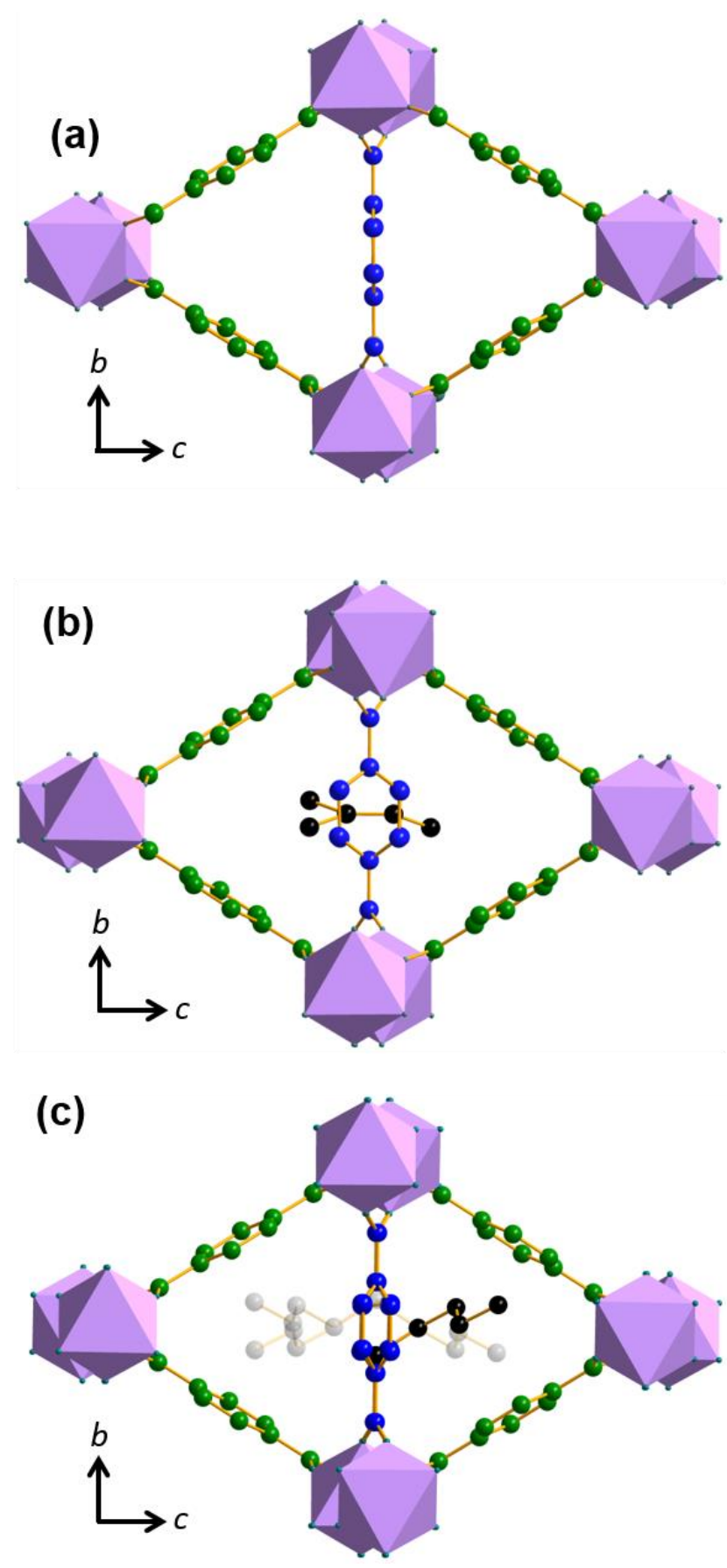

Figure 1. Structure of (a) orthorhombic $\mathrm{Sc}_{2} \mathrm{BDC}_{3}$ viewed parallel to the crystallographic $a$-axis, with $\mathrm{ScO}_{6}$ octahedra colored purple and $\mathrm{BDC}$ linkers colored by symmetry equivalence. $\mathrm{H}$ atoms are omitted for clarity. In (a), Groups 1 and 2 are colored green and blue, respectively. In (b) and (c), guest molecules are shown in black. For clarity, an ordered 2-methylbutane molecule is shown in (b). The $n$-pentane positions disordered by symmetry are shown in grey in (c).

(methane, ethane, propane; $\sim 4$ A). ${ }^{21}$ Crystallographic studies at low temperature show that methane and ethane fit closely into the available channel space of the $\mathrm{Sc}_{2} \mathrm{BDC}_{3}$, though adsorption sites for ethane are also observed in gaps in the channel walls between the aromatic rings. Preliminary packed column experiments indicated markedly shorter retention times over a packed column of $\mathrm{Sc}_{2} \mathrm{BDC}_{3}$ crystals for branched alkanes compared with normal alkanes of similar boiling points, suggesting greater uptakes of straight chain alkanes than their branched isomers. ${ }^{22}$ To determine whether this resulted from size exclusion, we investigated the adsorption behaviour of normal and branched alkanes up to saturated vapour pressures at room temperature and also at extreme pressures in a diamond anvil cell. The aim was to investigate whether guest uptake is limited to linear hydrocarbons, or if pressure could be used as a tool to overcome steric limitations. For this, we examined the adsorptive behaviour and associated structural response of $\mathrm{Sc}_{2} \mathrm{BDC}_{3}$ in the presence of a selection of hydrocarbons with varying chain lengths (from $\mathrm{C}_{5}$ to $\mathrm{C}_{8}$ ) and different degrees of branching.

\section{EXPERIMENTAL}

Synthesis. Single crystals of $\mathrm{Sc}_{2} \mathrm{BDC}_{3}$ were prepared solvothermally according to the literature. ${ }^{20}$ Thermogravimetric analysis confirmed the crystals contained no solvent in the pores prior to immersion and high-pressure diffraction experiments. Details of the synthesis and characterization are provided in the Supporting Information.

Gas adsorption isotherms. Gas isotherms for the different alkanes (2-methylbutane, $n$-pentane, $n$-hexane, 2,2-dimethylbutane, 2,3-dimethylbutane, $n$-heptane, 2-methylheptane and 2,2,4-trimethylpentane (Table 1)) were measured volumetrically at $298 \mathrm{~K}$ for $\mathrm{Sc}_{2} \mathrm{BDC}_{3}$ using a custom-built vacuum line. The $\mathrm{Sc}_{2} \mathrm{BDC}_{3}$ sample was degassed at $438 \mathrm{~K}$ under vacuum for $c a .2 \mathrm{~h}$ prior to measurement of each isotherm. The alkane was degassed using a repeated freeze/thaw process using liquid $\mathrm{N}_{2}$. The alkane uptake was determined as a function of increasing equilibrium pressure, up to pressures close to their saturated vapour pressures. A full description of the apparatus and method is given in the Supporting Information (Figures S3-S11).

In-situ immersion single crystal X-ray diffraction experiments. Single-crystal $\mathrm{X}$-ray diffraction experiments were performed on $\mathrm{Sc}_{2} \mathrm{BDC}_{3}$ crystals in a capillary, immersed in both $n$-pentane and 2-methylbutane, using a method described previously. ${ }^{23}$ A crystal was attached to the tip of a MiTeGen $100 \mu \mathrm{m}$ Microloop ${ }^{\mathrm{TM}}$ using a small amount of Araldite ${ }^{\mathrm{TM}}$ epoxy resin. The loop was mounted on a goniometer head and covered with a MicroRT ${ }^{\mathrm{TM}}$ polyester capillary. The capillary was stuck to the goniometer head and sealed around the base using Araldite ${ }^{\mathrm{TM}}$. When the epoxy resin was dry, the capillary was filled with the

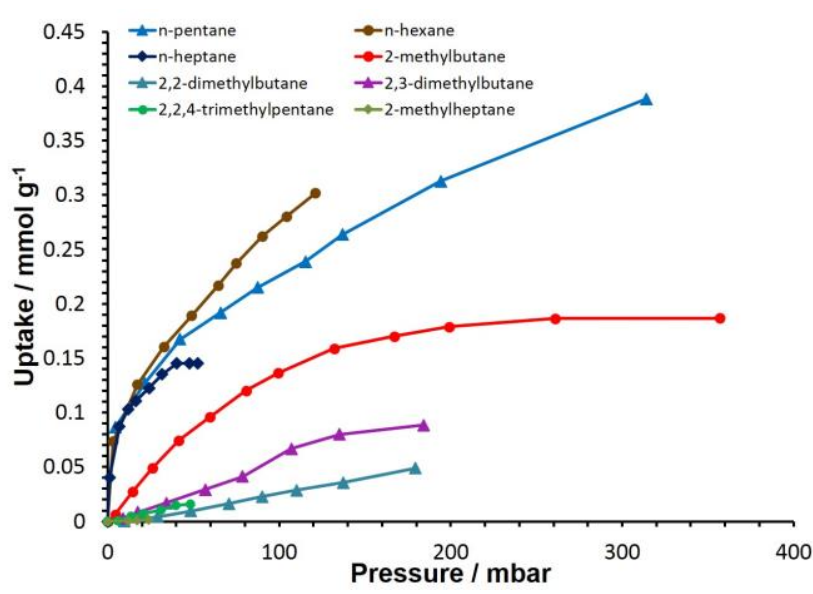

Figure 2. Adsorption isotherms for $\mathrm{Sc}_{2} \mathrm{BDC}_{3}$ for linear as well as monoand di-branched hydrocarbon species 
solvent in question (see Table 1) by injection through the top of

\begin{tabular}{|c|c|c|}
\hline Guest & Molecular structure & $\begin{array}{c}\text { Kinetic } \\
\text { diameter }(\AA)\end{array}$ \\
\hline$n$-pentane & & 4.5 \\
\hline 2-methylbutane & & 5.0 \\
\hline$n$-heptane & & 4.3 \\
\hline $\begin{array}{l}\text { 2-methyl } \\
\text { heptane }\end{array}$ & & 5.5 \\
\hline $\begin{array}{l}\text { 2,3-dimethyl } \\
\text { butane }\end{array}$ & & 6.0 \\
\hline $\begin{array}{l}\text { 2,2-dimethyl } \\
\text { butane }\end{array}$ & & \\
\hline $\begin{array}{l}\text { 2,2,4-trimethyl } \\
\text { pentane }\end{array}$ & & 6.2 \\
\hline
\end{tabular}

Table 1. Chemical structures and kinetic diameters of the linear and branched alkanes examined as guest species in $\mathrm{Sc}_{2} \mathrm{BDC}_{3}$ at low and high pressures.

the capillary using a $0.6 \mathrm{~mm}$ diameter needle. The resultant hole was sealed with a drop of molten beeswax. Diffraction data were then collected at room temperature, and processed as detailed in the Supporting Information.

High-pressure single crystal X-Ray diffraction. Highpressure single-crystal diffraction data were obtained by placing a single-crystal of $\mathrm{Sc}_{2} \mathrm{BDC}_{3}$ - together with a ruby chip for pressure calibration - in a Merril-Bassett Diamond Anvil Cell (DAC) with $600 \mu \mathrm{m}$ culet diamonds and a tungsten gasket. For each pressure series the gasket chamber was filled with a different solvent as hydrostatic medium and the DAC closed carefully. Diffraction data were then collected at room temperature and processed as detailed in the Supporting Information

Structure refinement. Structure solutions were performed using SUPERFLIP. ${ }^{24}$ In the cases where no satisfactory solution was obtained, the atomic coordinates of the previous pressure point were used as a starting model. The structures were refined against $F^{2}$ in CRYSTALS ${ }^{25}$ where thermal similarity and vibrational restraints were applied on all non-hydrogen atoms (except for $\mathrm{Sc}$ ) before refinement of anisotropic displacement parameters. Additional distance and angle restraints were also applied on the guest molecules and on the Group 2 BDC ring that is disordered as a consequence of the included guest. Hydrogen atoms attached to carbon atoms were placed geometrically and not refined. Analysis of the solvent accessible volume and the residual electron density using SQUEEZE ${ }^{26}$ was performed by applying the same resolution cut off of $1.1 \AA$ to each data set following the procedure described by Graham et $a l^{27}$. Further refinement details can be found in the Supporting Information. All crystallographic data is available from the CCDC 1410949-1410961.

DFT single-point energy calculations. The structure of $\mathrm{Sc}_{2} \mathrm{BDC}_{3}$ determined experimentally under ambient conditions (orthorhombic $F d d d$ ) was used as a starting model for the calculations. To save computational time the structure was transformed to its primitive cell. The energy of the primitive cell relative to the geometry-optimized global minimum was then calculated as a function of the angle $(\theta)$ of rotation around the 1,4phenylene axes of one Group 1 and one Group 2 linker (see Supporting Information, Figure S12). In both cases, calculations were performed with and without the TkatchenkoScheffler dispersion correction. Energy values, calculated in $10^{\circ}$ rotation increments, are provided in Table $\mathrm{S} 1$ in the supporting information.

\section{RESULTS AND DISCUSSION}

Immersion diffraction experiments and adsorption isotherms. The adsorption isotherms for alkanes on $\mathrm{Sc}_{2} \mathrm{BDC}_{3}$ obtained volumetrically at $298 \mathrm{~K}$ are shown in Figure 2. The straight chain alkanes $n$-pentane, $n$-hexane and $n$-heptane show strong uptake at low partial pressures $\left(0.14-0.18 \mathrm{mmol} \mathrm{g}^{-1}\right.$ at 40 mbar) indicating the molecules are taken up readily into the MOF. By contrast the geminal dimethylalkanes (2,2-dimethylbutane and 2,2,4-trimethylbutane) show very low uptakes $\left(<0.02 \mathrm{mmol} \mathrm{g}^{-1}\right.$ at $\left.40 \mathrm{mbar}\right)$. These low uptakes are not unexpected, considering the bulky t-butyl groups at the end of the molecules which must prevent these from penetrating into the pores of $\mathrm{Sc}_{2} \mathrm{BDC}_{3}$.

The mono-branched 2-methylbutane and the vicinal dimethyl 2,3-dimethylbutane uptakes are intermediate between $n$ - and geminal dibranched alkanes. In particular the 2-methylbutane shows considerable uptake. By contrast, 2-methylheptane shows very low uptake, possibly in part due to its low saturated vapour pressure at $298 \mathrm{~K}$.

In order to understand the uptake behaviour of $\mathrm{Sc}_{2} \mathrm{BDC}_{3}$ at low pressures and determine whether branched hydrocarbons can enter the pores under ambient conditions, we performed two immersion in situ single-crystal X-ray diffraction experiments,

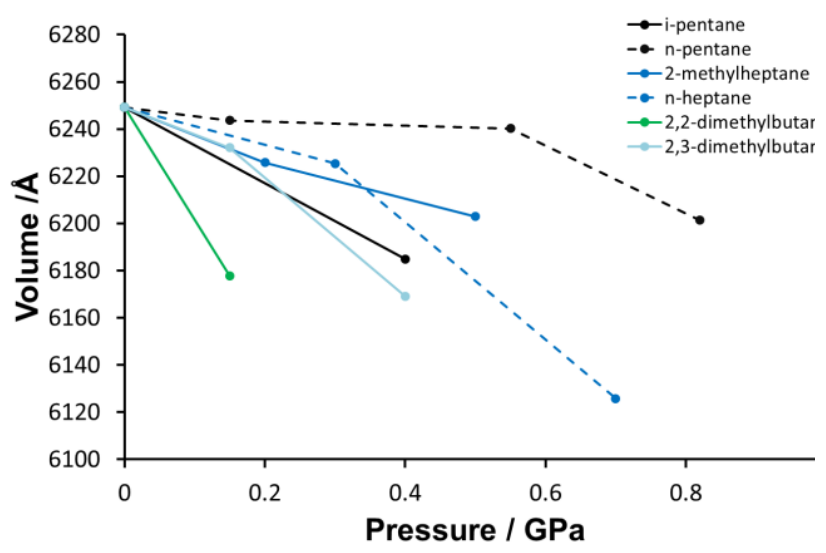

Figure 3. Unit cell volume as a function of pressure for the different hydrocarbons considered. Dotted lines refer to linear hydrocarbons whilst solid lines denote branched molecules. 


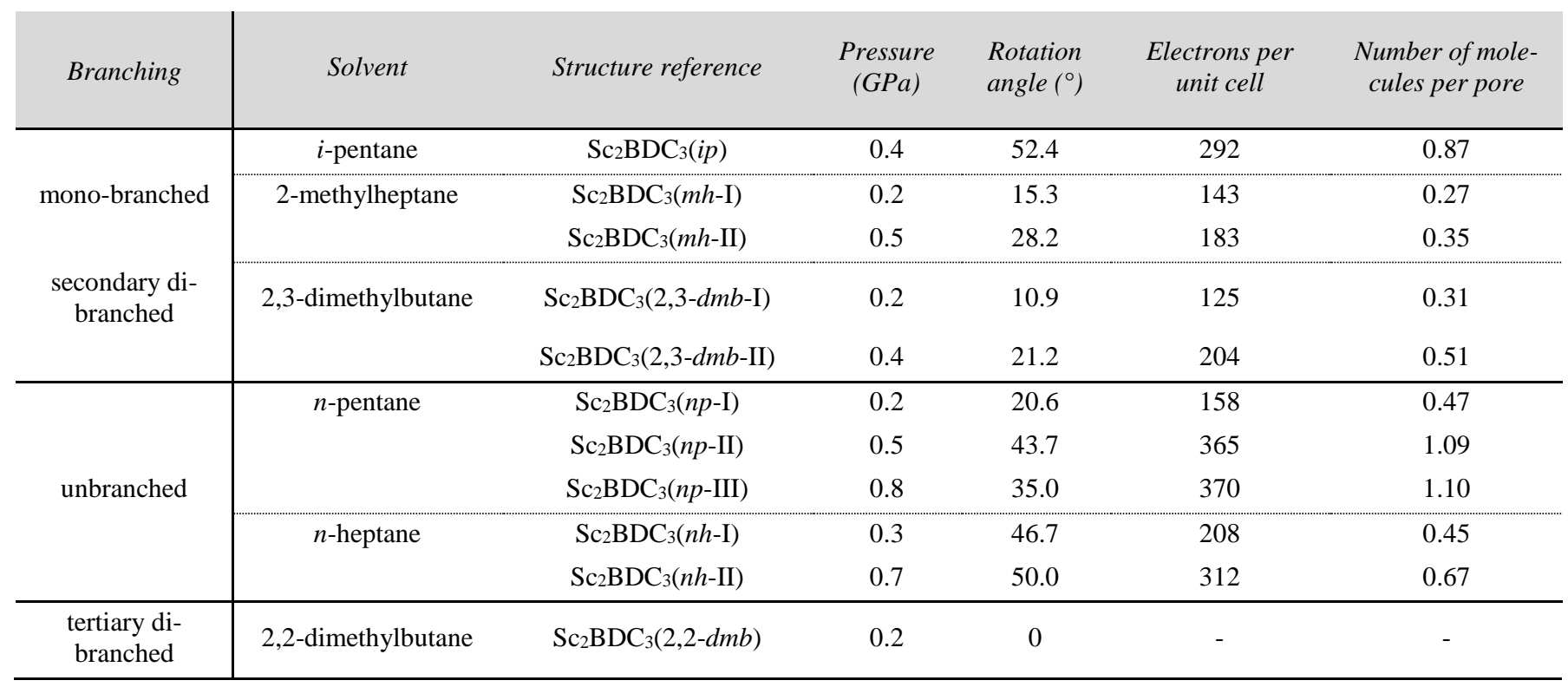

Table 2.Summary of rotation angle of BDC Group 2 linkers and pore content in the high-pressure $\mathrm{Sc}_{2} \mathrm{BDC}_{3} \mathrm{crystal}_{\mathrm{structures}}$ for various hydrocarbon guest species calculated from the PLATON SQUEEZE routine.

where a single-crystal of $\mathrm{Sc}_{2} \mathrm{BDC}_{3}$ was immersed in a linear alkane (n-pentane) and a branched alkane (2-methylbutane) and diffraction data collected in situ.

Immersion in $n$-pentane induced subtle structural changes to the framework structure. Although the adsorbed linear hydrocarbons could not be modelled crystallographically, a rotation of $14^{\circ}$ of the Group 2 BDC linkers was observed. Together with a slight increase in the residual electronic density in the pores, these immersion experiments suggest that upon soaking, $\mathrm{Sc}_{2} \mathrm{BDC}_{3}$ easily adsorbs linear hydrocarbons. By contrast, no crystallographic evidence for uptake of the branched and bulkier 2-methylbutane was obtained: when compared to the native $\mathrm{Sc}_{2} \mathrm{BDC}_{3}$ structure, neither structural changes in the framework nor significant increases in the residual electron density in the pores were observed. These observations expose the limitations of the in situ immersion diffraction experiments. These measurements have an inherently low sensitivity due to the very low uptake of guest species into the pores at ambient pressure and temperature making these experiments extremely challenging for modelling guest species. In order to increase uptake, a series of high-pressure single-crystal X-ray diffraction experiments was performed to see whether very high pressures could be used to force larger amounts of sterically hindered and oversized guest molecules into the pores.

High-pressure inclusion experiments. All alkanes listed in Table 1 were used as the pressure transmitting medium in high-pressures single crystal diffraction experiments on $\mathrm{Sc}_{2} \mathrm{BDC}_{3}$. In each case the pressure was increased from $c a .0 .2$ GPa until the samples became amorphous. While still crystalline, $\mathrm{Sc}_{2} \mathrm{BDC}_{3}$ was observed to retain its orthorhombic Fddd symmetry in all cases. The results of the variation of unit cell volume are given in Figure 3. The inclusion of molecules into MOFs is found not only to reduce the degree of unit cell contraction upon compression, but also to stabilize the frameworks against collapse. We have recently shown that the compression of $\mathrm{Sc}_{2} \mathrm{BDC}_{3}$ in methanol has little physical effect on the framework structure, but that the super-filling of methanol and guestguest interactions throughout the channels stabilizes the MOF at high pressures,${ }^{24}$ while similar behaviour has also been seen in other MOFs and zeolites. ${ }^{28}$

For the experiments using $n$-pentane, which is known to be readily adsorbed, there is strong resistance to compression and the structure remains crystalline until $0.8 \mathrm{GPa}$. By contrast, for 2,2,4-trimethylpentane, amorphization occurred immediately upon loading the cell at $0.2 \mathrm{GPa}$, and for the geminal 2,2-dimethylbutane the structure contracts strongly under pressure and loses crystallinity above $0.2 \mathrm{GPa}$, consistent with these molecules being too large to access the pores and stabilize the structure, even at elevated pressures. For all the other alkanes, the behaviour is intermediate, suggesting some pore filling occurs under pressure. For 2-methylbutane, when the pressure was increased beyond $0.4 \mathrm{GPa}$ the crystals became amorphous and underwent a change from colourless to black. In our previous high-pressure study on $\mathrm{Sc}_{2} \mathrm{BDC}_{3}$, we also reported this change of optical properties upon amorphization. When the crystals were removed from the DAC after releasing the pressure, in all cases the samples became colourless and fully crystalline again. This is consistent with our previous study in methanol.

High-pressure single crystal diffraction data allowed us not only to monitor how the framework responded to external pressure, but also to understand how the framework adapted to increasing guest content. When guest uptake had occurred, the extraframework electron density could be modelled by disordered alkane adsorbates. Some details of the measured crystal structures are given in Table 2, where the extra-framework electron density (modelled by using the SQUEEZE algorithm within PLATON) is given in terms of adsorbate molecules per pore. In line with the adsorption data, these results confirm that $n$-pentane is most readily taken up whilst 2,2-dimethylbutane is not adsorbed at all. As well as locating extra framework electron density in the pores, these single crystal structures show that the $\mathrm{Sc}_{2} \mathrm{BDC}_{3} \mathrm{MOF}$ can adapt to facilitate the uptake of alkanes through a ligand rotation.

This modification is best explained in terms of the starting empty structure. Under ambient temperature and pressure conditions, $\mathrm{Sc}_{2} \mathrm{BDC}_{2}$ crystallises in the orthorhombic space group $F d d d$, having two independent BDC linker molecules (Groups 

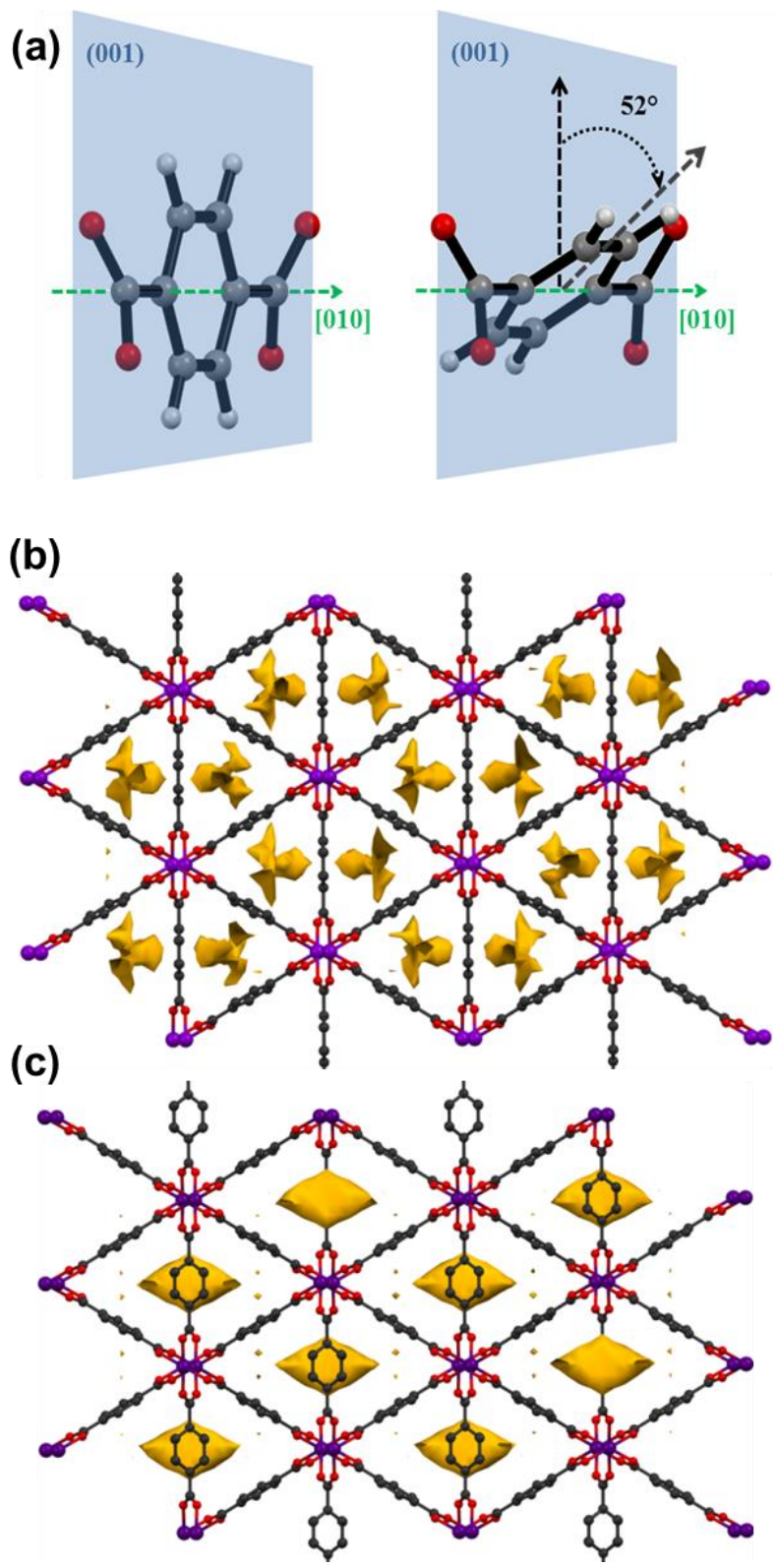

Figure 4. (a) Scheme of the ring rotation around [010] of Group 2 phenyl rings with respect to the (001) plane. On the left, the native $\mathrm{Sc}_{2} \mathrm{BDC}_{3}$ structure, on the right, the high-pressure guest-included structure $\mathrm{Sc}_{2} \mathrm{BDC}_{3}(i p)$. Only one of the statistically disordered rings is shown for clarity. (b) Solvent accessible surface (yellow) in one unit cell of $\mathrm{Sc}_{2} \mathrm{BDC}_{3}$. (c) Solvent accessible surface (yellow) in one unit cell of $\mathrm{Sc}_{2} \mathrm{BDC}_{3}(i p)$. 2-Methylbutane molecules are not shown in (c) to allow clear representation of the modified pore space.

1 and 2) the phenyl rings of which are parallel to the $a$-axis (i.e. parallel to the principal framework channel direction). Upon uptake of either linear or di-branched hydrocarbons, the Group 2 BDC rings are rotated around [010] into two statically disordered (50:50 occupancy) positions (Figure 4a).

This effect is most significant in the case of 2-methylbutane inclusion (henceforth referred to as $\mathrm{Sc}_{2} \mathrm{BDC}_{3}(i p)$; Figure $1 \mathrm{~b}$ and $4 \mathrm{c}$ ), where the rings rotate by $52.4^{\circ}$ (and $-52.4^{\circ}$ by symmetry) with respect to the (001) plane on which the Group 2 linkers lie in the empty $\mathrm{Sc}_{2} \mathrm{BDC}_{3}$ (Figure $4 \mathrm{a}$ ). Fddd symmetry is maintained. The phase transition has a profound effect on the pore size and shape. Sections of adjacent triangular channels - originally partitioned by the Group 2 BDC 'walls' in the parent $\mathrm{Sc}_{2} \mathrm{BDC}_{3}$ structure amalgamate to give eight rhomboidal cavities per unit cell in the high-pressure $\mathrm{Sc}_{2} \mathrm{BDC}_{3}(i p)$ phase (Figure $4 \mathrm{~b}, 4 \mathrm{c}$, and S13).

The intermolecular distance between adjacent Group 2 BDC linkers along the $a$-axis in empty $\mathrm{Sc}_{2} \mathrm{BDC}_{3}$ is $\sim 4.5 \AA(\mathrm{CH} \cdots \mathrm{HC}$; normalized values), whereas in $\mathrm{Sc}_{2} \mathrm{BDC}_{3}(\underline{i p})$ the distance between the two closest disordered BDC positions is $\sim 6.3 \AA$. This significantly increases the depth of the pores, allowing the bulky 2-methylbutane molecules to sit in the isolated pores between adjacent rotated rings along [100]. The pore volume ${ }^{29}$ indicates that the volume of each large pore in the high-pressure phase is $\sim 240 \AA^{3}$, compared with $\sim 137 \AA^{3}$ for each of the two equivalent sections of triangular channel in the ambient pressure structure that merge, allowing the bulky 2-methylbutane molecules to be incorporated into the framework (Figure $1 \mathrm{~b}$ and $4 c)$.

A comparison can be made between these high-pressure guestincluded $\mathrm{Sc}_{2} \mathrm{BDC}_{3}$ structures and the high pressure behaviour of ZIF-8. ${ }^{19}$ In ZIF-8, a pressure-induced ring rotation was observed when using methanol as a hydrostatic liquid at $1.5 \mathrm{GPa}$, causing the volume (and accessible pore volume) to increase with pressure. However, the overall accessible pore volume in the different high-pressure structures of $\mathrm{Sc}_{2} \mathrm{BDC}_{3}$ is actually reduced by around $10 \%$. Instead, the radical change in local pore structure shows that the ring rotation can be attributed to the shape of the guest molecule, not the requirement of the system to increase the pore volume.

Amorphization of the crystal in 2-methylbutane occurs above $0.4 \mathrm{GPa}$, substantially lower in pressure than in methanol. As the 2-methylbutane-bearing structure at $0.4 \mathrm{GPa}$ can no longer accommodate any more 2-methylbutane, it would appear that on increasing pressure further, direct compression and amorphization occurs for $\mathrm{Sc}_{2} \mathrm{BDC}_{3}(i p)$. $\mathrm{Sc}_{2} \mathrm{BDC}_{3}(i p)$ does not therefore experience the stabilizing effect of channel 'super-filling' which occurs with methanol, which also occurs in other MOFs with one, two and three-dimensional channel connectivity. ${ }^{15,23,30}$

Larger di-branched hydrocarbons can also penetrate into the pores of $\mathrm{Sc}_{2} \mathrm{BDC}_{3}$ at high pressures. Using 2,3-dimethylbutane and 2-methylheptane as hydrostatic media, a similar transformation is observed (see Figure 5a and b), though in each case the rotation is not as significant as in $\mathrm{Sc}_{2} \mathrm{BDC}_{3}(i p)$ : $21.2^{\circ}$ for 2,3-dimethylbutane and $28.2^{\circ}$ for 2-methylheptane. This can be explained by the amount of alkane that is being adsorbed into the framework. In the case of $\mathrm{Sc}_{2} \mathrm{BDC}_{3}$ (ip) a whole 2-methylbutane molecule is present in each pore at high pressure. On the other hand, at the highest pressure measured for both larger species $(0.4 \mathrm{GPa}$ for 2,3-dimethylbutane and $0.5 \mathrm{GPa}$ for 2methylheptane) the hydrocarbon uptake equates to less than half-occupancy for each guest molecule in the framework pores (see Table 2). For guests larger than 2-methylbutane a reduced uptake capacity results, which is coupled to a less substantial rotation angle of the Group 2 BDC ligands. This agrees well with trends observed in our gas adsorption experiments.

High-pressure diffraction experiments on $n$-pentane and $n$-heptane show a similar structural transition to that observed with mono-branched secondary hydrocarbons. However the shape of the molecules and their location within the cavity results in distinct differences in the structural behaviour of $\mathrm{Sc}_{2} \mathrm{BDC}_{3}$. These differences are particularly evident between 2-methylbutane and $n$-pentane. 

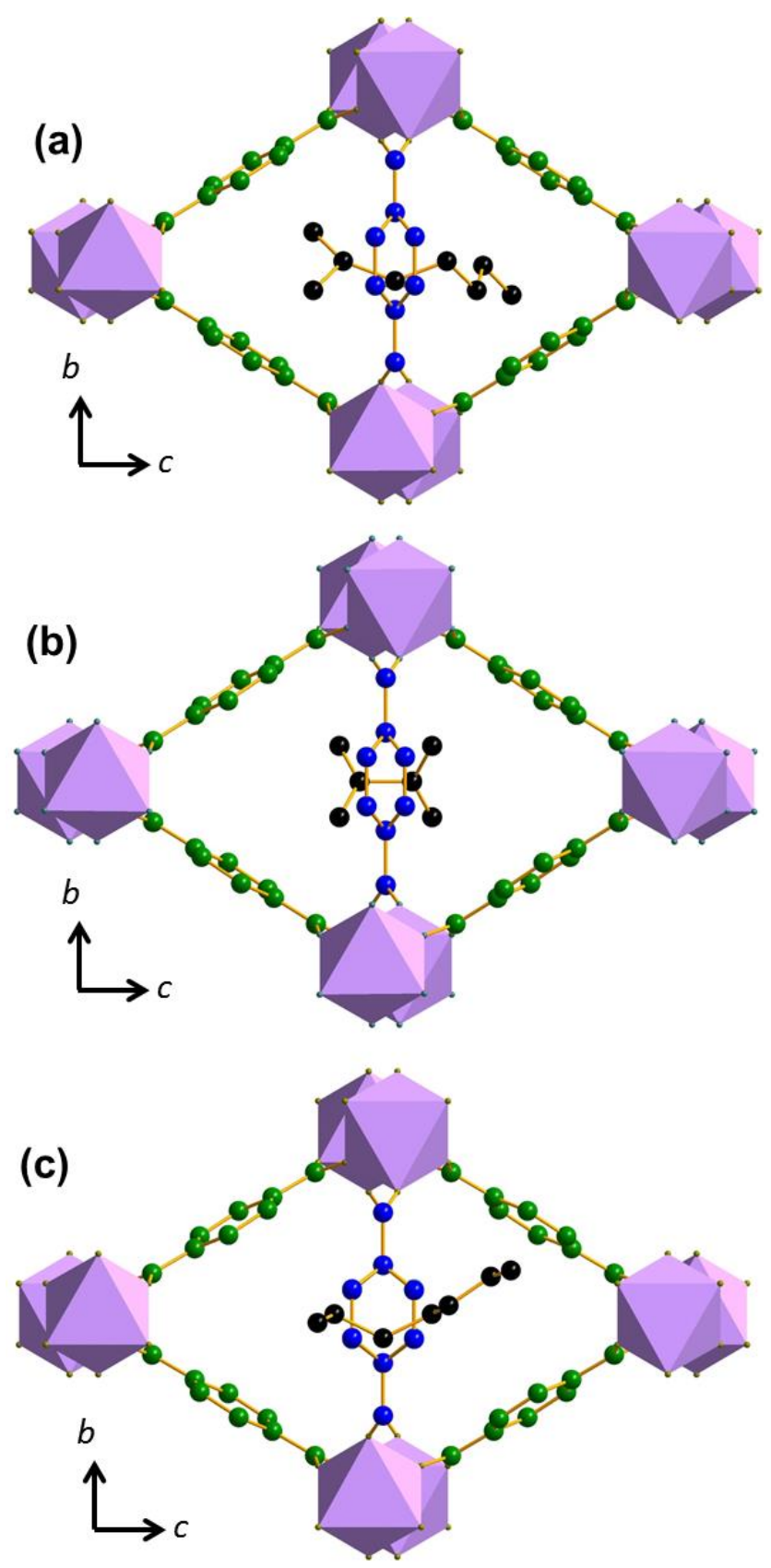

Figure 5. High-pressure crystal structures of $\mathrm{Sc}_{2} \mathrm{BDC}_{3}$ with the included guest molecules of (a) 2-methylheptane $\left(\mathrm{Sc}_{2} \mathrm{BDC}_{3}(m h\right.$-II)), (b) 2,3-dimethylbutane $\left(\mathrm{Sc}_{2} \mathrm{BDC}_{3}(2,3-d m b\right.$-II) $)$ and (c) $n$-heptane $\left(\mathrm{Sc}_{2} \mathrm{BDC}_{3}(n h\right.$-II). Ordered guest molecules are shown for clarity.

The longer chain and increased flexibility of $n$-pentane, in comparison to 2-methylbutane, causes the molecule to occupy additional adsorption sites, extending across both the existing channel space (along the [100] direction) and the deepened voids between the rotated BDC rings (as shown in Figure 1c). The longer, narrower shape of $n$-pentane results in three key differences between the structural response of $\mathrm{Sc}_{2} \mathrm{BDC}_{3}$ to $n$-pentane and 2-methylbutane uptake at high pressure. Firstly, the Group 2 BDC rings initially do not rotate to the same extent as in $\mathrm{Sc}_{2} \mathrm{BDC}_{3}(\mathrm{ip})$. There is slight variation over the pressure regime (from $20.8^{\circ}$ in $\mathrm{Sc}_{2} \mathrm{BDC}_{3}(n p-\mathrm{I})$ to $35.0^{\circ}$ in $\mathrm{Sc}_{2} \mathrm{BDC}_{3}$ (np-III)), but the rotation of $54.6^{\circ}$ in $\mathrm{Sc}_{2} \mathrm{BDC}_{3}(i p)$ is far more extreme. Secondly, the framework channels do not become 'closed' in $\mathrm{Sc}_{2} \mathrm{BDC}_{3}(n p-\mathrm{I})$, as is the case in $\mathrm{Sc}_{2} \mathrm{BDC}_{3}(i p)$. This is shown in Figure $\mathrm{S} 13$ and is a direct result of the lesser degree of BDC rotation in $\mathrm{Sc}_{2} \mathrm{BDC}_{3}(n p-\mathrm{I})$. Finally, and related wholly to the two previous points, the disordered sites of the $n$-pentane increase in occupancy as pressure is increased on the $\mathrm{Sc}_{2} \mathrm{BDC}_{3}$ crystal. Essentially, more $n$-pentane is forced into the framework as the pressure is increased, with a subsequent increase in rotation angle of the BDC linker. In our previous pressure study using methanol, an increase in occupancy of two disordered methanol adsorption sites could also be followed as a function of pressure, however no change in framework geometry occurred.

Inclusion of $n$-heptane yields similar effects to those observed with $n$-pentane. However in this case, the rotation angle recorded for both $n$-heptane included structures, $\mathrm{Sc}_{2} \mathrm{BDC}_{3}(h p-\mathrm{I})$ and $\mathrm{Sc}_{2} \mathrm{BDC}_{3}(h p$-II $)$ is more pronounced, measuring $46.7^{\circ}$ and $50.0^{\circ}$ respectively. This results from the longer chain length which extends not only along the channels but also between them. Comparing the uptake of $n$-heptane $(0.65$ molecules per pore) with that recorded for the monobranched 2-methylheptane ( 0.35 per pore) shows again the different behaviour of unbranched and branched hydrocarbons upon inclusion into $\mathrm{Sc}_{2} \mathrm{BDC}_{3}$.

Both in the case of $n$-pentane and $n$-heptane, crystallinity is maintained beyond $0.5 \mathrm{GPa}$, unlike in the case of branched hydrocarbons. With $n$-pentane it was possible to keep increasing the pressure without significant loss in the diffraction quality up until $0.8 \mathrm{GPa}$. Amidst a gradual loss of resolution, it is not until $1.7 \mathrm{GPa}$ that amorphization is complete, however, only unit cell dimensions could be obtained above $0.8 \mathrm{GPa}$. As mentioned previously, in both $\mathrm{Sc}_{2} \mathrm{BDC}_{3}(n p$-II $)$ and $\mathrm{Sc}_{2} \mathrm{BDC}_{3}(n p$-III) the occupancy of the included $n$-pentane molecules gradually increases to $100 \%$, and coincides with the Group 2 BDC rotation angle increase, resulting in a single molecule of $n$-pentane per rhomboidal pore. Thus, the possibility to continue filling the framework results in a delay in the amorphization, showing a markedly different behaviour between the compression with branched and unbranched hydrocarbons.

Using 2,2-dimethylbutane or 2,2,4-trimethylpentane as hydrostatic media, no evidence of guest uptake was detected in $\mathrm{Sc}_{2} \mathrm{BDC}_{3}$. Whilst for 2,2-dimethylbutane the only high-pressure crystal structure collected at $0.2 \mathrm{GPa}\left(\mathrm{Sc}_{2} \mathrm{BDC}_{3}(2,2-\mathrm{dmb})\right)$, revealed no obvious structural change. Additionally, the unit cell volume was substantially reduced; a clear indication of direct framework compression in tertiary branched hydrocarbons.

This indicates that tertiary branched alkanes are too big to enter the framework pores, even at high pressures, and that the secondary di-branched hydrocarbons represent the upper diameter limit for a guest species under pressure in $\mathrm{Sc}_{2} \mathrm{BDC}_{3}$. This in principle could enable $\mathrm{Sc}_{2} \mathrm{BDC}_{3}$ to act as a molecular sieve between secondary and tertiary branched alkanes under pressure. The results show that the very high pressures in the DAC are required to achieve high adsorbate loadings of branched alkanes in $\mathrm{Sc}_{2} \mathrm{BDC}_{3}$. At these pressures, significant $P \Delta V$ work is available to give rise to rotation of phenyl groups of the linker and to maintain them in a rotated, 'high energy', state, to allow hydrocarbon uptake. At $0.1 \mathrm{GPa}$ this is of the order of $10 \mathrm{~kJ} \mathrm{~mol}^{-}$ ${ }^{1}$, for example, increasing linearly with pressure. To calculate the energy associated with the rotation of Group 2 linker phenyl groups, we performed density functional theory single-point en- 
ergy calculations of the potential energy of rotation in $10^{\circ}$ rotation increments (Table S1 and Figure 6). For observed rotations in the $10-50^{\circ}$ range, energies of $5-20 \mathrm{~kJ} \mathrm{~mol}^{-1}$ of linker are observed, which could readily be offset by the work performed on the included hydrocarbons in the GPa regime would make a significant contribution.

It is also of interest to understand why it is the Group 2 linker phenyl groups, and not those of the Group 1 linker, that rotate, especially when the adsorption of $\mathrm{CO}_{2}$ is observed to cause a minor tilt of the Group 1 rings while the Group 2 ring remains unperturbed. Comparison of the rotation energies (Figure 6) indicates that there is a higher energy required for significant rotation of the Group 1 linkers. Consideration of the steric limitations of different BDC groups indicates that concerted rotation of equivalent and adjacent Group 1 linkers (to a greater degree than the minor rotation already reported) could result in unfavourably close edge-to-edge contacts $(<1.25 \AA)$ between aromatic rings. The Group $2 \mathrm{BDC}$ has more degrees of freedom to rotate since all adjacent BDC linkers are Group 1. The larger Tkatchenko-Scheffler dispersion correction term for Group 2, especially at higher rotation angles, also indicates that there may be additional van der Waals stabilization, which is not the case for the Group 1 linkers

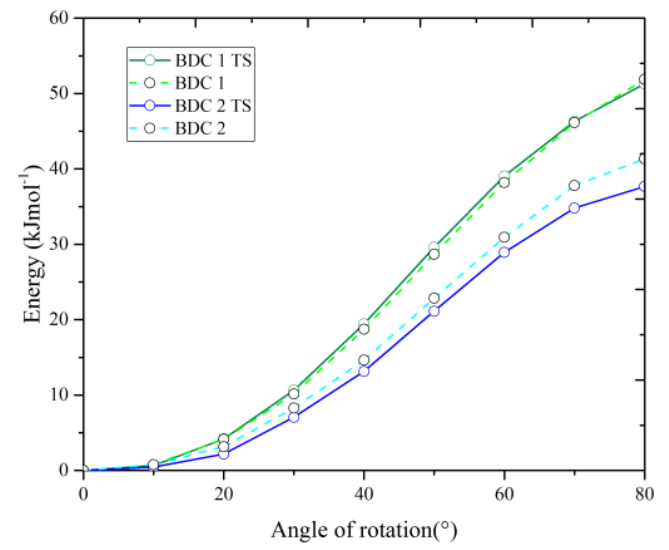

Figure 6. Relative unit cell energy as a function of rotational angle of Group 1 (green squares) and Group 2 (blue circles) BDC linkers. Solid lines denote values corrected with the Tkatchenko-Scheffler (TS) dispersion correction and dotted lines denote values without the correction.

\section{CONCLUSIONS}

In summary, we have demonstrated how sterically 'oversized' hydrocarbon molecules, can be squeezed into accessible cavities of the small-pore $\mathrm{MOF}, \mathrm{Sc}_{2} \mathrm{BDC}_{3}$, using high pressure. Since $\mathrm{C}_{5}-\mathrm{C}_{8}$ hydrocarbons cannot penetrate or diffuse through the framework in the same way as shorter unbranched hydrocarbons, inclusion results in a structural phase transition which alters the shape and size of the individual pores with relatively little change to the overall porosity. As a result of the ring rotation, guest molecules of an awkward shape are able to be admitted. Since this transition is fully reversible (no damage, fractures or polycrystallinity is imparted on the crystal), this result is akin to 'breathing' mechanisms in frameworks, exemplifying how high pressure X-ray diffraction can be used to probe the structural flexibility of MOFs, and is a powerful tool for understanding the structural effect of guest uptake. The framework responds differently to inclusion of linear, mono-branched, secondary di-branched or tertiary branched hydrocarbons and so this material may have application in alkane separations. For instance, mono-branched hydrocarbons can penetrate the framework void space under relatively moderate pressures, while tertiary branched hydrocarbons cannot. Similarly, only linear molecules are readily adsorbed under ambient conditions. Such a potential application of $\mathrm{Sc}_{2} \mathrm{BDC}_{3}$ in the high-pressure separation of alkane isomers would be facilitated by the complete reversibility of the adsorption/desorption process.

\section{ASSOCIATED CONTENT}

\section{AUTHOR INFORMATION}

\section{Corresponding Author}

*s.moggach@ed.ac.uk

Notes

The authors declare no competing financial interests

\section{Author Contributions}

The work was performed through contributions of all authors. All authors have given approval to the final version of the manuscript.

\section{ACKNOWLEDGMENT}

We thank the Royal Society of Edinburgh and the Scottish Government for a fellowship to Dr Stephen A. Moggach. We thank EPSRC $(\mathrm{EP} / \mathrm{J} 02077 \mathrm{X} / 1)$ and Leverhulme Trust for a research project grant (RPG-209) for financial support. We also thank the UK Carr Parinello consortium for allocation of computing time on the EPSRC high performance computing resource ARCHER (managed by the Edinburgh Parallel Computing Centre, the EaSTCHEM Research Computing Facility and the University of Edinburgh ECDF facility. The data which underpin this work are available at http://dx.doi.org/10.7488/ds/371.

\section{REFERENCES}

(1) Farrusseng, D. Metal-organic frameworks : applications from catalysis to gas storage; Wiley-VCH: Weinheim, 2011.

(2) Li, J.-R.; Sculley, J.; Zhou, H.-C. Chemical Reviews 2011, 112, 869.

(3) Herm, Z. R.; Wiers, B. M.; Mason, J. A.; van Baten, J. M.; Hudson, M. R.; Zajdel, P.; Brown, C. M.; Masciocchi, N.; Krishna, R.; Long, J. R. Science 2013, 340, 960.

(4) Wu, D.; Maurin, G.; Yang, Q.;

Serre, C.; Jobic, H.; Zhong, C. J. Mater. Chem. A 2014, 2, 1657.

(5) Chen, L.; Morrison, C. A.; Duren, T. J. Phys. Chem. C 2012, 116, 18899.

(6) Chen, L.; Grajciar, L.; Nachtigall, P.; Duren, T. J. Phys. Chem. C 2011, 115, 23074. (7) Loiseau, T.; Serre, C.; Huguenard, C.; Fink, G.; Taulelle, F.; Henry, M.; Bataille, T.; Ferey, G. Chemistry-a European Journal 2004, 10, 1373. 
(8) Serre, C.; Millange, F.;

Thouvenot, C.; Nogues, M.; Marsolier, G.;

Louer, D.; Ferey, G. Journal of the American

Chemical Society 2002, 124, 13519.

(9) Chen, L.; Mowat, J. P. S.; Fairen-

Jimenez, D.; Morrison, C. A.; Thompson, S. P.;

Wright, P. A.; Duren, T. J. Am. Chem. Soc. 2013, 135, 15763.

(10) Haldoupis, E.; Nair, S.; Sholl, D. S. J. Am. Chem. Soc. 2010, 132, 7528.

(11) Bux, H.; Liang, F.; Li, Y.;

Cravillon, J.; Wiebcke, M.; Caro, J. Journal of

the American Chemical Society 2009, 131, 16000.

(12) Bux, H.; Chmelik, C.; van Baten,

J. M.; Krishna, R.; Caro, J. Advanced Materials 2010, 22, 4741.

(13) Coudert, F.-X.; Boutin, A.; Fuchs,

A. H. Mol. Phys. 2014, 112, 1257.

(14) Fairen-Jimenez, D.; Moggach, S. A.; Wharmby, M. T.; Wright, P. A.; Parsons, S.;

Dueren, T. Journal of the American Chemical

Society 2011, 133, 8900.

(15) Graham, A. J.; Allan, D. R.;

Muszkiewicz, A.; Morrison, C. A.; Moggach, S.

A. Angewandte Chemie-International Edition

2011, 50, 11138.

(16) Coudert, F.-X. Chem. Mater.

2015, 27, 1905.

(17) Zhou, W.; Wu, H.; Hartman, M.

R.; Yildirim, T. Journal of Physical Chemistry C 2007, 111, 16131.

(18) Huang, X. C.; Lin, Y. Y.; Zhang,

J. P.; Chen, X. M. Angewandte Chemie-

International Edition 2006, 45, 1557.

(19) Moggach, S. A.; Bennett, T. D.;

Cheetham, A. K. Angewandte Chemie-

International Edition 2009, 48, 7087.
(20) Miller, S. R.; Wright, P. A.; Serre, C.; Loiseau, T.; Marrot, J.; Ferey, G. Chemical Communications 2005, 3850.

(21) Miller, S. R.; Wright, P. A.; Devic, T.; Serre, C.; Ferey, G.; Llewellyn, P. L.; Denoyel, R.; Gaberova, L.; Filinchuk, Y.

Langmuir 2009, 25, 3618.

(22) Mowat, J. P. S., University of St. Andrews, 2012.

(23) McKellar, S. C.; Graham, A. J.; Allan, D. R.; Mohideen, M. I. H.; Morris, R. E.; Moggach, S. A. Nanoscale 2014, 6, 4163.

(24) Palatinus, L.; Chapuis, G. Journal of Applied Crystallography 2007, 40, 786.

(25) Betteridge, P. W.; Carruthers, J.

R.; Cooper, R. I.; Prout, K.; Watkin, D. J. Journal of Applied Crystallography 2003, 36, 1487.

(26) van der Sluis, P.; Spek, A. L. Acta Crystallographica Section A 1990, 46, 194.

(27) Graham, A. J.; Allan, D. R.; Muszkiewicz, A.; Morrison, C. A.; Moggach, S. A. Angew Chem Int Ed Engl 2011, 50, 11138.

(28) Coasne, B.; Haines, J.; Levelut, C.; Cambon, O.; Santoro, M.; Gorelli, F.; Garbarino, G. Phys. Chem. Chem. Phys. 2011, 13, 20096.

(29) Spek, A. L.; Utrecht University: Utrecht, The Netherlands, 2004.

(30) Graham, A. J.; Tan, J.-C.; Allan, D. R.; Moggach, S. A. Chem. Commun. 2012, 48, 1535 .

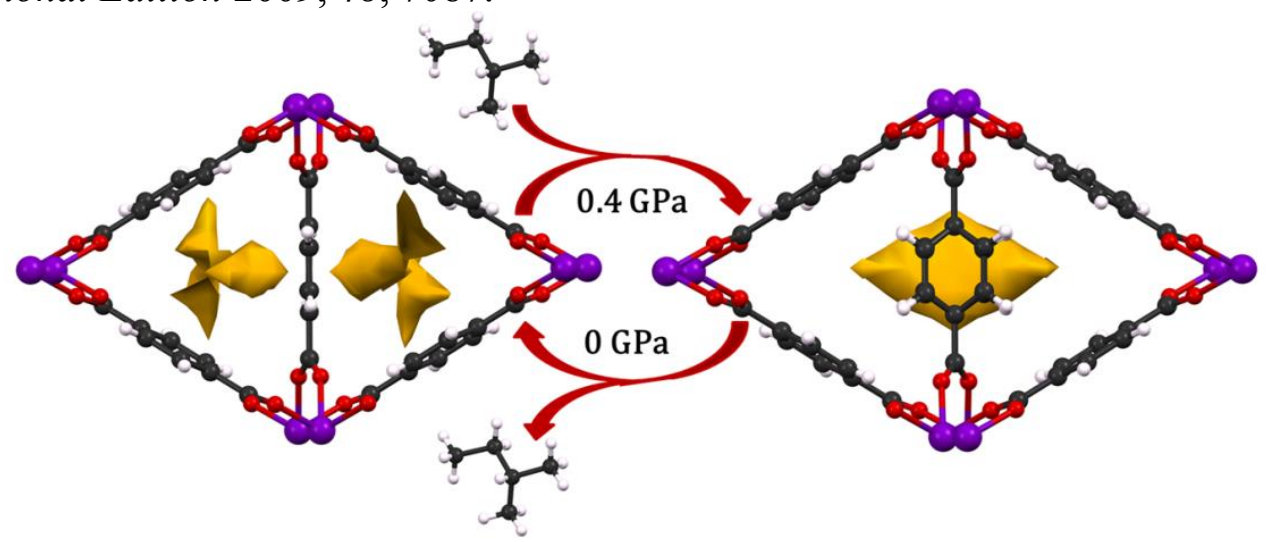

